\title{
Predictors of response to palliative care intervention for chronic nausea in advanced cancer outpatients
}

\author{
Wadih Rhondali • Sriram Yennurajalingam • \\ Gary Chisholm • Jeanette Ferrer • Sun Hyun Kim • \\ Jung Hun Kang • Marilene Filbet • Eduardo Bruera
}

Received: 13 September 2012 / Accepted: 25 March 2013 /Published online: 16 April 2013

(C) Springer-Verlag Berlin Heidelberg 2013

\begin{abstract}
Purpose Nausea is a frequent and distressing symptom in advanced cancer patients. The objective of this retrospective study was to determine predictors of response to palliative care consultation for chronic nausea in advanced cancer outpatients.

Methods Eligible patients included were outpatient supportive care center seen consecutively for an initial consultation and who had one follow-up visit within 30 days of the initial consultation. We reviewed the medical records of 1,273 consecutive patients, and $444(35 \%)$ were found to meet
\end{abstract}

W. Rhondali $\cdot$ S. Yennurajalingam $\cdot$ J. Ferrer $\cdot$ S. H. Kim $\cdot$

J. H. Kang $\cdot$ E. Bruera $(\square)$

Department of Palliative Care and Rehabilitation Medicine,

Unit 141, University of Texas MD Anderson Cancer Center,

1515 Holcombe Boulevard,

Houston, TX 77030, USA

e-mail: ebruera@mdanderson.org

W. Rhondali $\cdot$ M. Filbet

Department of Palliative Care, Centre Hospitalier de Lyon-Sud, Hospices Civils de Lyon, 164 chemin du Grand Revoyet, 69495 Pierre Bénite Cedex,

Lyon, France

G. Chisholm

Department of Biostatistics, Unit 1414,

University of Texas MD Anderson Cancer Center,

1515 Holcombe Boulevard,

Houston, TX 77030, USA

\section{S. H. Kim}

Department of Family Medicine, Myong Ji Hospital, Kwandong University, College of Medicine,

Gyeonggi, South Korea

\section{J. H. Kang}

Department of Internal Medicine, Institute of Health Science, College of Medicine, Gyeongsang National University,

Jinju, South Korea the eligibility criteria. All patients were assessed using the Edmonton Symptom Assessment Scale (ESAS). Nausea response was defined as an improvement of at least $30 \%$ between the initial visit and the first follow-up. We used logistic regression models to assess the possible predictors of improvement in nausea.

Results Overall, 112 of 444 patients (25\%) experienced moderate/severe chronic nausea (ESAS item score $\geq 4 / 10$ ). Higher baseline nausea intensity was significantly related to constipation $(r=0.158 ; p=0.046)$ and all the symptoms assessed by the ESAS $(p<0.001)$. Sixtyeight of the $112(61 \%)$ patients with moderate/severe nausea at baseline showed a significant improvement at the follow-up visit $(p<0.001)$. The main predictors for nausea response were improvement of fatigue $(p=0.005)$ and increased appetite $(p=0.003)$.

Conclusions Baseline nausea was associated with all the ESAS symptom and improvement of fatigue and lack of appetite predicted a lower frequency of nausea at follow-up. More research is necessary to better understand the association between nausea severity and other symptoms and to predict which interventions will yield the best outcomes depending on the mix and severity of symptoms.

Keywords Palliative care $\cdot$ Chronic nausea $\cdot$ Symptom management

\section{Introduction}

Patients with advanced cancer experience notable physical and psychosocial symptoms $[1,2]$. The most frequent symptoms include pain, fatigue, weight loss, lack of appetite, nausea, anxiety, shortness of breath, and confusion [3]. The main causes of these symptoms are related to progression of the disease and complications of treatments such as 
chemotherapy and/or radiation therapy. Symptom distress is a significant predictor of quality of life in advanced cancer patients [4]. Among these patients, the frequency of one particularly distressing symptom, chronic nausea, is approximately $60 \%$ [5].

Nausea may be defined as an unpleasant feeling of the need to vomit, often accompanied by autonomic symptoms such as pallor, cold sweat, salivation, tachycardia, and diarrhea [5]. The etiology of chronic nausea in advanced cancer patients is often multifactorial. It can be caused by drugs such as opioids, delayed gastric emptying, mechanical bowel obstruction, increased intracranial pressure, vestibular dysfunction, metabolic problems, and cortical effects such as anxiety or depression. The most common cause is delayed gastric emptying (in $35-44 \%$ of cases) $[6,7]$. In addition, symptoms such as chronic nausea have been reported to be closely related to other symptoms and to patient demographics [8].

Chronic nausea has been ranked as the most distressing side effect of chemotherapy [9]. The management of this stressful multifactorial symptom requires comprehensive interdisciplinary care because the negative consequences of leaving it uncontrolled can include physical complications of anorexia and fatigue, impaired performance status, diminished quality of life, and increased medical costs and psychosocial distress [10]. Failure to control nausea may also further decrease a cancer patient's chances of prolonged survival or cure if the nausea causes delays in treatment [11]. Although few studies have looked at the epidemiology of chronic nausea, its intensity and the predictors of response to treatment determining these features would aid on developing appropriate management strategies regarding this symptom.

Palliative care services have been shown to be effective in managing many distressing symptoms in advanced cancer patients [6, 12-15]; however, predictive factors for response remain unclear. Our objective in this retrospective study was to determine predictors of response to outpatient palliative care consultation for chronic nausea. We reasoned that if this study gave us the ability to stratify patients into risk categories for chronic nausea, then we would be able to develop optimal treatment strategies in patients with advanced cancer and thereby improve their quality of life.

\section{Methods}

\section{Participants}

This study received approval from The University of Texas MD Anderson Cancer Center Institutional Review Board. We reviewed the electronic medical records of consecutive patients seen by palliative care physicians in the outpatient
Supportive Care Center for an initial consultation between February 18, 2008 and February 18, 2010. Patients were included if they were 18 years or older and had a diagnosis of advanced cancer (defined as locally advanced, recurrent or metastatic disease) and were referred to the Supportive Care Clinic. To be eligible for this study, patients had to have prospectively completed the Edmonton Symptom Assessment Scale (ESAS) questionnaire at the initial and the follow-up clinic visits and have attended at least one followup visit within 30 days of the initial consultation.

\section{Supportive care clinic}

The Supportive Care Center's interdisciplinary team is led by board-certified physicians. Other important members include a palliative care-trained registered nurse, pharmacist, nutritionist, chaplain, social worker, psychiatric nurse-counselor, and wound care nurse. The care of all patients follows a standardized management plan [16]. Patients and their families are initially assessed by the registered nurse using tools such as the ESAS, Memorial Delirium Assessment Scale (MDAS), constipation and family support questionnaires [2]. Patients are routinely screened by nurses for constipation, asking them about their usual bowel movement, the consistency of the stools and their last bowel movement. The nurse then discusses the findings with a palliative care specialist, who subsequently conducts interviews the patient and the family and performs a physical examination. The physician and the nurse then ask appropriate members of the interdisciplinary team to participate based on the patient's and family's particular needs. These interventions and care provided by the interdisciplinary team follow palliative care guidelines established by the National Comprehensive Cancer Network and National Consensus Project and have been outlined elsewhere [17]. They focus on (a) assessing and managing cancerrelated symptoms, including pain, fatigue, nausea, anorexia, anxiety, depression, sedation, dyspnea, sleep disturbance, and impaired feeling of well-being; (b) facilitating patients' and caregivers' understanding of the disease and treatment goals; and (c) providing assistance to patients and their caregivers in coping with a life-threatening illness and in decision-making.

\section{Instruments}

The ESAS measures the response to ten common symptoms (pain, fatigue, nausea, depression, anxiety, drowsiness, shortness of breath, lack of appetite, sleep disorders, and impaired feeling of well-being) in patients with cancer. The ESAS asks patients to rate the intensity of these symptoms over the past $24 \mathrm{~h}$ using an 11-point numeric rating scale, from 0 (no symptoms) to 10 (worst possible symptoms) [18-20]. This questionnaire has been validated in cancer populations [19, 21, 22] and has been reported as a good tool for nausea assessment in 
this population [23]. The ESAS yields a total score and two subscale scores. These scores have been validated in advanced cancer patients with good reliability when compared to others tools $[19,20,22,24]$. The total symptom distress score (TSDS) is the sum of the scores for the ten symptoms for a total score of $0-100$ [22]. The physical distress subscore (PHS) was the sum of scores for seven symptoms (pain, nausea, tiredness or fatigue, drowsiness, appetite, shortness of breath or dyspnea, and sleep), and the psychological distress subscore (PSS) was the sum of scores for three symptoms (depression, anxiety, and feeling of well-being) [25].

The CAGE questionnaire is a simple, four-item, validated tool used to screen for a recent history of alcoholism; this questionnaire is already incorporated in our palliative care service's routine assessment [26]. A finding of two positive answers is more than $85 \%$ sensitive and $90 \%$ specific for the diagnosis of alcohol abuse and/or dependence [27]. Patients positive for alcoholism are at risk for higher symptom distress [28].

\section{Assessment}

Demographic information (i.e., age, sex, ethnicity) and clinical data (i.e., cancer diagnosis, primary symptom diagnosis, metastatic site) were collected. We documented chemotherapy regimen (agent, dose) as well as radiation (irradiated site, dose per fraction) during the previous month. We classified the type of chemotherapy regarding the potential emetogenicity using the American Society of Clinical Oncology (ASCO), European Society for Medical Oncology (ESMO) and Multinational Association Of Supportive Care In Cancer (MASCC) guidelines $[29,30]$. We also documented radiation during the previous month (irradiated sites and dose per fraction). We classified the risk level of the irradiated sites using the ESMO and MASCC guidelines [30]. Lastly, we documented cancerrelated surgery during the previous month.

We also collected scores from the ESAS, a constipation assessment, information regarding any current bowel obstructions, signs of current increased intracranial pressure, the CAGE questionnaire, the type of opioid and the morphine equivalent daily dose (MEDD), The total dose each analgesic was translated into its oral MEDD using standard tables [31].

We also reviewed the medication regimen (e.g., antiemetics, laxatives) received by the patient at the time of the referral to the palliative care team as well as the medication changes after the consult.

We also collected data on interventions (pharmacologic or non-pharmacologic) provided by the palliative care team by reviewing clinical/consultation notes. The outcomes of these interventions were evaluated by quantifying symptom evolution between the initial consultation and the first follow-up. Interventions were determined by reviewing the consultation notes completed by the palliative care team.
We defined clinical response as an improvement of at least $30 \%$ or more on the ESAS between the initial visit and the first follow-up visit, as suggested by others [32-34].

\section{Statistical considerations}

We report categorical variables with frequencies and percentages and continuous variables by their mean and standard deviation if they were normally distributed. If they were not normally distributed, we report the median and interquartile range (Q1-Q3), and analyzed the data using non-parametric methods. Student's two-tailed $t$-test was used to compare the frequencies of no/mild nausea and moderate/severe nausea. Assuming alpha $=0.05$, we calculated that we would have $>99 \%$ power to detect a difference of least $30 \%$ on the ESAS scale [35]. Similarly, each individual factor from all of the instruments was tested for any effect on the ESAS score using Student's two-tailed $t$-test. Because there were many comparisons, we corrected the results using Bonferroni methods. Power was estimated to remain $>99 \%$ for up to 50 comparisons. Pearson correlation coefficients and associated $\mathrm{p}$ values are reported comparing each ESAS item between no/mild and moderate/severe nausea. Mann-Whitney $U$-tests and chisquare tests were performed to determine factors associated with chronic nausea, where chronic nausea is defined as patients with at least moderate nausea (scores $\geq 4 ; N=112$ ). Estimates of effect size are presented as odds ratios (OR) based on the results of univariate logistic regressions. Stepwise logistic regression was attempted to create a multivariate model for change in ESAS nausea item score following palliative care consultation. As no model retained more than one significant variable, multiple regression results are not reported in this paper. We considered $p$ values $<0.001$ statistically significant. All statistical analyses were performed using SAS version 9.2 (SAS Inc., Cary, NC).

\section{Results}

\section{Patient characteristics}

Of the 1,273 consecutive screened patients, 444 (35\%) met the eligibility criteria. Among eligible patients, the median age was 59 years (Q1-Q3, 51-65) and 218 (49\%) were women (Table 1). The most common types of cancer were gastrointestinal and respiratory. Fifty patients $(11 \%)$ had brain metastases. During the preceding month prior to palliative consultation, 183 patients ( $46 \%$ ) received chemotherapy and $83(45 \%)$ patients received moderately to highly emetogenic chemotherapy. Sixty (14\%) received radiotherapy during the month preceding their initial consultation with $24(41 \%)$ patients receiving dose per fraction higher than $3 \mathrm{~Gy}$, and four (7\%) patients receiving irradiation in a 
Table 1 Patient characteristics $(N=444)$

\begin{tabular}{|c|c|}
\hline Patient characteristics & $N(\%)$ \\
\hline Male & $226(51)$ \\
\hline Age (years), median (Q1-Q3) & $59(51-65)$ \\
\hline \multicolumn{2}{|l|}{ Ethnicity } \\
\hline White & $326(73)$ \\
\hline African American & $60(14)$ \\
\hline Asian/Pacific Islander & $22(5)$ \\
\hline Other & $36(8)$ \\
\hline \multicolumn{2}{|l|}{ Cancer diagnosis } \\
\hline Breast & $50(11)$ \\
\hline Gastrointestinal & $98(22)$ \\
\hline Genitourinary & $41(9)$ \\
\hline Gynecologic & $35(8)$ \\
\hline Head and neck & $65(15)$ \\
\hline Hematologic & $24(5)$ \\
\hline Respiratory & $75(17)$ \\
\hline Other $^{\mathrm{a}}$ & $56(13)$ \\
\hline \multicolumn{2}{|l|}{ Metastasis $^{\mathrm{b}}$} \\
\hline Brain & $50(11)$ \\
\hline Liver & $121(27)$ \\
\hline Bone & $146(33)$ \\
\hline Other & $330(74)$ \\
\hline \multicolumn{2}{|l|}{ Cancer treatment the previous month } \\
\hline Chemotherapy & $183(46)$ \\
\hline \multicolumn{2}{|l|}{ Degree of emetogenicity (incidence) } \\
\hline Minimal $(<10 \%)$ & $23(13)$ \\
\hline Low $(10-30 \%)$ & $77(42)$ \\
\hline Moderate (30-90\%) & $23(13)$ \\
\hline High $(>90 \%)$ & $60(32)$ \\
\hline Radiotherapy & $60(14)$ \\
\hline \multicolumn{2}{|l|}{ Risk level according to irradiated sites } \\
\hline Minimal & $3(5)$ \\
\hline Low & $52(88)$ \\
\hline Moderate & $4(7)$ \\
\hline Dose per fraction $>3 \mathrm{~Gy}$ & $24(41)$ \\
\hline Concomitant chemo-radiotherapy & $25(6)$ \\
\hline Other specific therapy (phase $1 / 2$ treatment) & $30(7)$ \\
\hline Surgery & $4(0.01)$ \\
\hline
\end{tabular}

Q1-Q3 first through third quartiles

${ }^{a}$ These include neuroendocrine cancers, sarcoma, melanoma, skin cancers, brain cancers, and unknown malignancies

${ }^{\mathrm{b}}$ The total is higher than $100 \%$ because some patients had multiple sites of metastasis. MEDD: mean equivalent daily dose; Q1-Q3: first through third quartiles

moderate risk site and non in high risk site. Only 25 (6\%) patients received concomitant chemo-radiotherapy and four $(0.01 \%)$ had surgery in the same period (Table 1$)$.
Baseline chronic nausea intensity and associated outcomes

Table 2 shows that of the 444 patients, $112(25 \%)$ experienced moderate/severe chronic nausea (ESAS score for nausea $\geq 4 / 10$ ) at baseline, with a median score for the complete sample of 1 (Q1-Q3, 0-4). Two hundred twenty-two patients $(50 \%)$ were constipated and $280(63 \%)$ were receiving opioids with a median MEDD of $90 \mathrm{mg}$ (Q1-Q3, 48-180).

We did not find any association between patient demographic, clinical characteristics or the treatment received (chemotherapy, radiotherapy) during the month preceding their initial consultation and the intensity of baseline nausea (Table 3). However, higher baseline nausea intensity was significantly related to constipation

Table 2 Clinical characteristics of patients with at least one follow-up visit $(N=444)$

Variables $N(\%)$

Symptom intensity, median (Q1-Q3)

Pain

$6(3-8)$

Fatigue

$6(4-8)$

Nausea

$1(0-4)$

Depression

$2(0-5)$

Anxiety

$3(0-5)$

Shortness of breath

$2(0-5)$

Appetite

$5(3-7)$

Drowsiness

$4(1-6)$

Sleep

$5(3-8)$

Well-being

$5(4-7)$

CAGE $(>1)$

$57(13)$

Baseline nausea intensity, median (Q1-Q3)

$1(0-4)$

Follow-up nausea intensity

None (0)

$216(49)$

Mild (1-3) 116(26)

Moderate (4-6) 68 (15)

Severe (7-10) $44(10)$

Constipation $222(50)$

Median time between the 2 visits (days), 15 (11-21) median (Q1-Q3)

Opioids $280(63)$

Opioids MEDD (mg), median (Q1-Q3) 90 (48-180)

Antiemetics $^{\mathrm{a}} 159(36)$

- Metoclopramide 55 (12)

- Ondansetron $97(22)$

- Others (prochlorperazine, promethazine, 64 (14) granisetron patch)

$\begin{array}{ll}\text { Corticoids } & 81 \text { (18) }\end{array}$

${ }^{\mathrm{a}}$ The total is higher than $100 \%$ because some patients had multiple antiemetic treatment 
Table 3 Association between baseline nausea and the other symptoms and medications $(N=444)$

ESAS Edmonton Symptom Assessment Scale, PSS psychological distress subscore, TSDS total symptom distress score, $P H S$ physical distress subscore, $Q 1-$ Q3 first through third quartiles, $S D$ standard deviation

*Pearson correlation

\begin{tabular}{|c|c|c|c|}
\hline Variables & $\begin{array}{l}\text { No nausea/mild } \\
\text { nausea }(<4) N=332\end{array}$ & $\begin{array}{l}\text { Moderate/severe } \\
\text { nausea }(\geq 4) N=112\end{array}$ & $r ; p^{*}$ \\
\hline \multicolumn{4}{|l|}{ ESAS item, median score (Q1-Q3) } \\
\hline Pain & $5(3-8)$ & $7(4-9)$ & $r=0.176 ; p<0.001$ \\
\hline Fatigue & $6(4-8)$ & $7(6-9)$ & $r=0.296 ; p<0.001$ \\
\hline Depression & $2(0-5)$ & $4(1-6)$ & $r=0.291 ; p<0.001$ \\
\hline Anxiety & $2(0-5)$ & $5(2-7)$ & $r=0.274 ; p<0.001$ \\
\hline Drowsiness & $3(0-5)$ & $5(3-8)$ & $r=0.297 ; p<0.001$ \\
\hline Shortness of breath & $2(0-5)$ & $4(0-6)$ & $r=0.219 ; p<0.001$ \\
\hline Lack of appetite & $5(2-7)$ & $6(5-9)$ & $r=0.287 ; p<0.001$ \\
\hline Sleep disturbance & $5(2-7)$ & $6(4-8)$ & $r=0.199 ; p<0.001$ \\
\hline Impaired feeling of well-being & $5(3-7)$ & $7(5-8)$ & $r=0.288 ; p<0.001$ \\
\hline PSS & $9(5-14)$ & $15(9-20)$ & $r=0.562 ; p<0.001$ \\
\hline PHS & $26(20-34)$ & $40(34-47)$ & $r=0.582 ; p<0.001$ \\
\hline TSDS & $36(27-46)$ & $56(46-65)$ & $r=0.562 ; p<0.001$ \\
\hline \multicolumn{4}{|l|}{ Other symptoms and medications } \\
\hline Constipation, $N(\%)$ & $153(46)$ & $69(62)$ & $r=0.158 ; p=0.046$ \\
\hline Chemotherapy the last month & $135(74)$ & $48(26)$ & $r=0.004 ; p=0.599$ \\
\hline Radiation the last month & $45(75)$ & $15(25)$ & $r=-0.002 ; 0.966$ \\
\hline Concomitant chemo-radiotherapy & $18(72)$ & $7(28)$ & $r=0.016 ; p=0.743$ \\
\hline Opioid MEDD, median (Q1-Q3) & $90(45-185)$ & $96(60-180)$ & $r=0.001 ; p=0.988$ \\
\hline Anti-emetic medication, $N(\%)$ & $101(30)$ & $58(52)$ & $r=0.194 ; p<0.001$ \\
\hline Metoclopramide, $N(\%)$ & $35(11)$ & $20(18)$ & $r=0.096 ; p=0.042$ \\
\hline Corticoids, $N(\%)$ & $61(18)$ & $20(18)$ & $r=-0.006 ; p=0.903$ \\
\hline Laxatives, $N(\%)$ & $158(48)$ & $70(63)$ & $r=0.116 ; p=0.015$ \\
\hline
\end{tabular}

$(r=0.158 ; p=0.046)$ and all the symptoms assessed by the $\operatorname{ESAS}(p<0.001)$. We also found a significant positive association between baseline nausea intensity and the presence of anti-emetic medication $(r=0.193$; $p<0.001)$; laxative use was significantly related to higher nausea intensity $(r=0.116 ; p<0.015)$ as well as metoclopramide $(r=0.096 ; p=0.042)$.

Chronic nausea intensity at the follow-up visit and associated outcomes

Table 4 shows no significant difference in the ESAS nausea response based on the moderate severe category of baseline nausea. We found that 156 patients (35\%) experienced no change in their nausea intensity between the two visits and $145(33 \%)$ and $143(32 \%)$ experiencing a worsening and improvement in nausea intensity, respectively.

Table 5 shows no univariate association between baseline ESAS, medication changes and other symptoms and ESAS nausea response.

ESAS nausea improvement after one visit to our supportive care outpatient center was associated with improvements in fatigue, and appetite at follow-up (Table 6).
Among the 112 patients with moderate/severe nausea, 69 (62\%) also experienced constipation at baseline compared to $33(29 \%)$ at the follow-up visit. Of the 69 constipated patients at baseline, 44 were not constipated anymore at the follow-up visit. Of these 44 patients, 36 (82\%) had a $\geq 30 \%$ improvement in nausea, about the twice the rate among patients who were constipated at both the baseline and the follow-up visit.

Table 4 Distribution of baseline ESAS nausea score within ESAS nausea response ${ }^{\mathrm{a}}$ to treatment category $(N=112)$

\begin{tabular}{llll}
\hline $\begin{array}{l}\text { Baseline nausea } \\
\text { intensity }\end{array}$ & $\begin{array}{l}\text { Non-responders } \\
N=44\end{array}$ & $\begin{array}{l}\text { Responders }^{\mathrm{a}} \\
N=68\end{array}$ & $p^{\mathrm{b}}$ \\
\hline $\begin{array}{l}\text { Moderate nausea (4-6), } \\
N=68\end{array}$ & $27(40)$ & $41(60)$ & 0.910 \\
$\begin{array}{l}\text { Severe nausea (7-10), } \\
N=44\end{array}$ & $17(39)$ & $27(61)$ & \\
\hline
\end{tabular}

${ }^{a}$ Responder defined as $30 \%$ improvement on ESAS nausea item score at follow-up for patients with baseline moderate/severe nausea. Nonresponder defined as less than $30 \%$ improvement on ESAS nausea item score at follow-up for patients with baseline moderate/severe nausea

${ }^{\mathrm{b}}$ Chi-square test 
Table 5 Baseline ESAS, medications and other baseline symptoms by nausea response (univariate; $N=112$ )
${ }^{a}$ Responder defined as $30 \%$ improvement on ESAS nausea item score at follow-up for patients with baseline moderate/severe nausea

${ }^{\mathrm{b}}$ Mann-Whitney $U$-test

${ }^{\mathrm{c}} \mathrm{Chi}$-square test

\begin{tabular}{lccll}
\hline Variable at baseline & $\begin{array}{l}\text { Non-responders } \\
N=44\end{array}$ & $\begin{array}{l}\text { Responders } \\
N=68\end{array}$ & $p$ & OR (95\% CI) \\
\hline Pain & $7(5-9)$ & $6.5(4-8)$ & 0.3358 & $0.93(0.81-1.06)$ \\
Fatigue & $7(6-8.5)$ & $7(6-8.5)$ & 0.6925 & $0.94(0.78-1.14)$ \\
Nausea & $5.5(4.5-7)$ & $6(5-7)$ & 0.5325 & $1.08(0.85-1.37)$ \\
Depression & $4(2-6)$ & $3(1-6.5)$ & 0.9402 & $0.99(0.88-1.12)$ \\
Anxiety & $5(2.5-7)$ & $4(1-7)$ & 0.9402 & $0.95(0.84-1.07)$ \\
Lack of appetite & $7(5-8.5)$ & $6(5-9)$ & 0.3769 & $0.97(0.85-1.12)$ \\
Drowsiness & $5.5(3.5-7)$ & $5(3.5-8)$ & 0.6245 & $1.03(0.90 .1 .20)$ \\
Impaired feeling of well-being & $7(5-8.5)$ & $7(4.5-8)$ & 0.1973 & $0.86(0.73-1.02)$ \\
Shortness of breath & $4.5(0.5-7)$ & $3(0-6)$ & 0.3595 & $0.95(0.84-1.07)$ \\
Sleep disturbance & $7(5-8)$ & $6(3-8.5)$ & 0.2257 & $0.89(0.77-1.04)$ \\
PSS & $15(11.5-19)$ & $12.5(8-20)$ & $0.219^{\mathrm{b}}$ & $0.97(0.92-1.03)$ \\
PHS PSS & $42.5(35.5-47)$ & $40(33-47.5)$ & $0.331^{\mathrm{b}}$ & $0.98(0.94-1.02)$ \\
TSDS PSS & $57(48.5-67)$ & $54(44-64)$ & $0.250^{\mathrm{b}}$ & $0.98(0.96-1.01)$ \\
Constipation N (\%) & $23(52 \%)$ & $46(68 \%)$ & $0.102^{\mathrm{c}}$ & $1.91(0.88-4.16)$ \\
Anti-emetic medication & $37(84 \%)$ & $49(72 \%)$ & $0.141^{\mathrm{c}}$ & $0.49(0.19-1.28)$ \\
$\quad$ (started or increased at & & & & \\
$\quad$ consult visit) N (\%) & $31(70 \%)$ & $38(56 \%)$ & $0.122^{\mathrm{c}}$ & $0.53(0.24-1.19)$ \\
Metoclopramide medication & & & & \\
$\quad$ (started or increased at & & $100(60-180)$ & $0.869^{\mathrm{c}}$ & $0.999(0.996-1.001)$ \\
$\quad$ consult visit) N (\%) & $90(60-135)$ & &
\end{tabular}

At the follow-up visit, 68 (61\%; $95 \%$ confidence interval $[\mathrm{CI}], 56-66 \%)$ of the 112 patients experiencing moderate/severe nausea at baseline showed a significant improvement $(p<0.001)$; however, because other patients developed or had worsening nausea, the total number of patients experiencing moderate/severe nausea at follow-up was the same as that at baseline (Fig. 1).

Treatment change after the initial consult visit

The most frequent pharmacological intervention was the initiation/increase of laxative use in $54 \%$ of patients, followed by initiation/increase of opioids and anti-emetics (Fig. 2). Regarding antiemetic medications, we found an important increase of the number of metoclopramide prescription from 54 (12\%) to 196 (44\%).

\section{Discussion}

In this study, we found that nausea was a frequent symptom, with 228 patients $(51 \%)$ experiencing at least some nausea and 112 patients $(25 \%)$ experiencing moderate/severe nausea. Our findings suggest that constipation was significantly more frequent in patients with moderate/severe nausea. In addition, the results suggest that constipation management was a key step in nausea management, as shown by the high rate of nausea abatement among patients who were initially
Table 6 Associations of response ${ }^{\mathrm{a}}$ of ESAS nausea to treatment with response $\mathrm{e}^{\mathrm{b}}$ of other ESAS items (univariate; $N=112$ )

${ }^{a}$ Responder defined as $30 \%$ improvement on ESAS nausea item score at follow-up for patients with baseline moderate/severe nausea

${ }^{\mathrm{b}}$ Thirty percent improvement on ESAS item score at follow-up

\begin{tabular}{llll}
\hline ESAS response $^{\mathrm{b}}$ & Odds ratio & $95 \% \mathrm{CI}$ & $P$ \\
\hline Pain & 1.74 & $(0.71-4.25)$ & 0.2265 \\
Fatigue & 4.53 & $(1.58-13.00)$ & 0.0049 \\
Depression & 2.12 & $(0.95-4.73)$ & 0.0670 \\
Anxiety & 1.90 & $(0.86-4.21)$ & 0.1114 \\
Lack of appetite & 3.41 & $(1.52-7.64)$ & 0.0029 \\
Drowsiness & 3.03 & $(1.11-8.23)$ & 0.0298 \\
Impaired feeling of well-being & 2.67 & $(1.18-6.03)$ & 0.0185 \\
Shortness of breath & 2.53 & $(1.08-5.93)$ & 0.0331 \\
Sleep disturbance & 1.38 & $(0.63-3.01)$ & 0.4159 \\
\hline
\end{tabular}


Fig. 1 Nausea intensity at first follow-up according to baseline nausea intensity

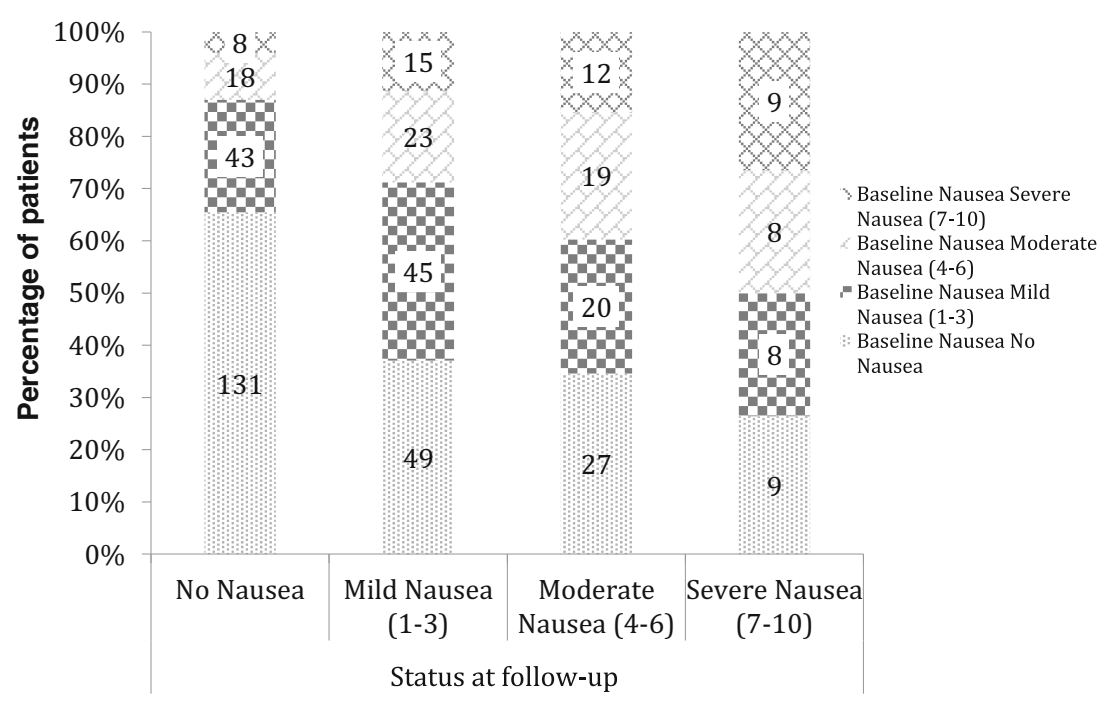

constipated (82\%) compared with patients who were not constipated at baseline (50\% and $51 \%$ ). The link between constipation and nausea is often reported in palliative care literature $[36,37]$ but very few studies have been able to demonstrate this association. On the basis of these findings, we previously suggested that as part of managing nausea, one should systematically screen patients for constipation [38]. However, larger prospective studies are needed to validate these findings and test the utility of such screening.

The frequency of chronic of nausea reported in this study is consistent with results reported in previous studies [37, 39, 40]; however, compared to previous studies, which explored nausea related to chemotherapy [17, 34, 41], our study is one of the first to evaluate the specific problem of chronic nausea among outpatient advanced cancer patients receiving palliative care.
This study also confirms our previous finding that even one outpatient palliative care consultation can have a positive effect on many cancer-related symptoms. Specifically the response rate for nausea of $61 \%$ supports the $71 \%$ rate of improvement in our previous study. [2] These results were also consistent with prior published literature [37, 42]. Nausea responded better to intervention than either fatigue or a poor feeling of well-being [43]. Response in our patients was probably related to changes in the antiemetic regimen (i.e., metoclopramide) as well as the overall symptom management.

We believe these results are relevant because palliative care specialists are highly trained in the assessment and management of symptoms and because nausea assessment was done prospectively using validated tools in advanced cancer patients. Primary teams should take advantage of these results
Fig. 2 Most frequent pharmacological interventions by nature of the intervention $(N=444)$. NSAID non-steroidal anti-inflammatory drug

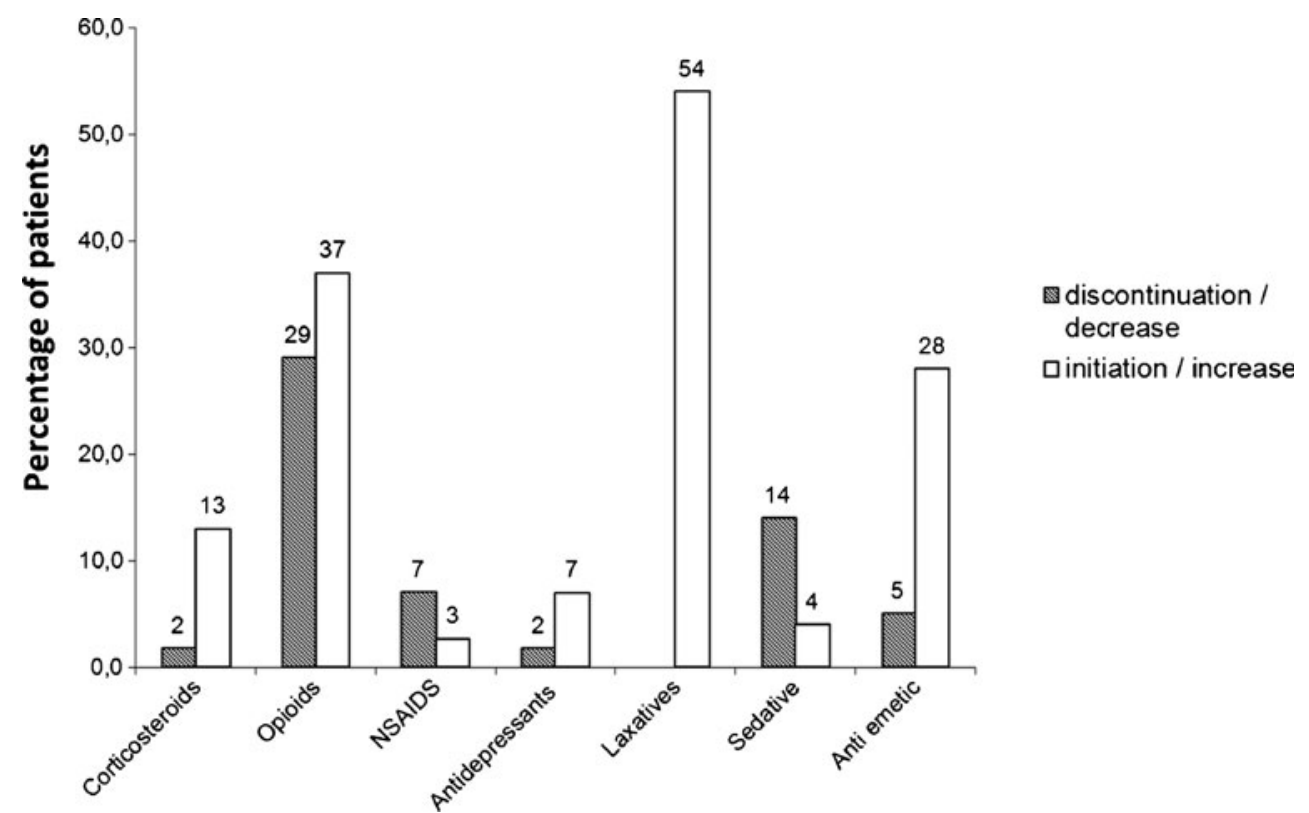


(e.g., early and frequent use of metoclopramide, constipation management and overall symptom management) to improve chronic nausea relief.

The most frequent pharmacological intervention was the initiation/increase of laxative use in $54 \%$ of patients. These results suggest that laxatives were probably under-used in these patients prior to their palliative care consultations. Assessment and management of constipation has been one of the basic skills taught by palliative care specialists since the beginning of the palliative care movement [44].

We did not find any significant predictors associated with nausea response in our multivariate analysis, suggesting that attempts to identify a single cause in palliative care patients often reveal multiple causes, as reported in the literature [45]. The only significant associated factors for nausea response were improvements in other ESAS symptoms such as anorexia and fatigue (Table 6), suggesting that symptom management in this frail population cannot be achieved by managing a single symptom (nausea) but requires using a multi-symptom approach.

Our results also show that although some patients experienced an improvement in nausea, about the same number experienced worsening. The latter group of patients might benefit from closer follow-up or routine phone assessments to improve the management of their symptoms.

Our work had several limitations. For example, because this study was retrospective, we encountered difficulties in capturing all the events that might have interacted with nausea management. One other limitation is the way constipation was assessed (with a yes/no question). Our team recently reported that constipation should be assessed using an 11-point numeric rating scale of 0 (no symptoms) to 10 (worst possible symptoms)[38]. That type of assessment would probably have allowed us to better comprehend the link between nausea and constipation. Another limitation of the study is the lack of control group or lack of patients in the setting other than seen by outpatient palliative care. It is possible that similar improvement in nausea could be observed by follow-up in a regular oncology clinic. Hence, further prospective controlled studies are needed. More research is also necessary to better understand the association between the severity of nausea and that of other symptoms in cancer patients and to better associate interventions with outcomes. Research that includes prospective longitudinal studies will help to characterize nausea and develop optimal treatment strategies on the basis of risk and response.

Acknowledgments Preparation of this manuscript is supported in part by the. MD Anderson Cancer Center support grant CA 016672; American Cancer Society (RSG-11-170-01-PCSM) [S.Y] and National Institutes of Health grants R01NR010162-01A1, R01CA1222292.01, and R01CA124481-01[E.B].We thank Ms. Kathryn Carnes and Ms. Amelia Scholtz for manuscript review.
Conflict of interest The authors made no disclosures.

\section{References}

1. Walsh D, Rybicki L (2006) Symptom clustering in advanced cancer. Support Care Cancer 14(8):831-836

2. Yennurajalingam S, Urbauer DL, Casper KLB, Reyes-Gibby CC, Chacko R, Poulter V, Bruera E (2011) Impact of a palliative care consultation team on cancer-related symptoms in advanced cancer patients referred to an outpatient supportive care clinic. J Pain Symptom Manage 41(1):49-56

3. Walsh D, Donnelly S, Rybicki L (2000) The symptoms of advanced cancer: relationship to age, gender, and performance status in 1,000 patients. Support Care Cancer 8(3):175-179

4. McMillan SC, Small BJ (2002) Symptom distress and quality of life in patients with cancer newly admitted to hospice home care. Oncol Nurs Forum 29(10):1421-1428

5. Davis MP, Walsh D (2000) Treatment of nausea and vomiting in advanced cancer. Support Care Cancer 8(6):444-452

6. Stephenson J, Davies A (2006) An assessment of aetiology-based guidelines for the management of nausea and vomiting in patients with advanced cancer. Support Care Cancer 14(4):348-353

7. Harris DG (2010) Nausea and vomiting in advanced cancer. Br Med Bull 96:175-185

8. Cheung W, Le L, Gagliese L, Zimmermann C (2011) Age and gender differences in symptom intensity and symptom clusters among patients with metastatic cancer. Support Care Cancer 19(3):417-423

9. Coates A, Abraham S, Kaye SB, Sowerbutts T, Frewin C, Fox RM, Tattersall MH (1983) On the receiving end-patient perception of the side-effects of cancer chemotherapy. Eur J Cancer Clin Oncol 19(2):203-208

10. Pirri C, Katris P, Trotter J, Bayliss E, Bennett R, Drummond $P$ (2011) Risk factors at pretreatment predicting treatment-induced nausea and vomiting in Australian cancer patients: a prospective, longitudinal, observational study. Support Care Cancer 19(10):1549-1563

11. Richardson JL, Marks G, Levine A (1988) The influence of symptoms of disease and side effects of treatment on compliance with cancer therapy. J Clin Oncol 6(11):1746-1752

12. Bruera E, Michaud M, Vigano A, Neumann CM, Watanabe S, Hanson J (2001) Multidisciplinary symptom control clinic in a cancer center: a retrospective study. Support Care Cancer 9(3):162-168

13. Bentley A, Boyd K (2001) Use of clinical pictures in the management of nausea and vomiting: a prospective audit. Palliat Med 15(3):247-253

14. Strasser F, Sweeney C, Willey J, Benisch-Tolley S, Palmer JL, Bruera E (2004) Impact of a half-day multidisciplinary symptom control and palliative care outpatient clinic in a comprehensive cancer center on recommendations, symptom intensity, and patient satisfaction: a retrospective descriptive study. J Pain Symptom Manage 27(6):481-491

15. Delgado-Guay MO, Parsons HA, Li Z, Palmer LJ, Bruera E (2009) Symptom distress, interventions, and outcomes of intensive care unit cancer patients referred to a palliative care consult team. Cancer 115(2):437-445

16. Elsayem A BE (2008) The M.D. Anderson symptom control and palliative care handbook. The University of Texas Health Science Center, Houston

17. McNicholl MP, Dunne K, Garvey A, Sharkey R, Bradley A (2006) Using the Liverpool Care Pathway for a dying patient. Nurs Stand 20(38):46-50 
18. Bruera E, MacMillan K, Hanson J, MacDonald RN (1989) The Edmonton staging system for cancer pain: preliminary report. Pain 37(2):203-209

19. Chang VT, Hwang SS, Feuerman M (2000) Validation of the Edmonton Symptom Assessment Scale. Cancer 88(9):2164-2171

20. Richardson LA, Jones GW (2009) A review of the reliability and validity of the Edmonton Symptom Assessment System. Curr Oncol 16(1):55

21. Carvajal A, Centeno C, Watson R, Bruera E (2011) A comprehensive study of psychometric properties of the Edmonton Symptom Assessment System (ESAS) in Spanish advanced cancer patients. Eur J Cancer 47(12):1863-1872

22. Bruera E, Kuehn N, Miller MJ, Selmser P, Macmillan K (1991) The Edmonton Symptom Assessment System (ESAS): a simple method for the assessment of palliative care patients. J Palliat Care 7(2):6-9

23. Hamling $\mathrm{K}$ (2011) The management of nausea and vomiting in advanced cancer. Int J Palliat Nurs 17(7):321-327

24. Moro C, Brunelli C, Miccinesi G, Fallai M, Morino P, Piazza M, Labianca R, Ripamonti C (2006) Edmonton symptom assessment scale: Italian validation in two palliative care settings. Support Care Cancer 14(1):30-37

25. Cheung WY, Barmala N, Zarinehbaf S, Rodin G, Le LW, Zimmermann C (2009) The association of physical and psychological symptom burden with time to death among palliative cancer outpatients. J Pain Symptom Manage 37(3):297304

26. Ewing JA (1984) Detecting alcoholism. The CAGE questionnaire. JAMA 252(14):1905-1907

27. Liskow B, Campbell J, Nickel EJ, Powell BJ (1995) Validity of the CAGE questionnaire in screening for alcohol dependence in a walk-in (triage) clinic. J Stud Alcohol 56(3):277-281

28. Parsons HA, Delgado-Guay MO, El Osta B, Chacko R, Poulter V, Palmer JL, Bruera E (2008) Alcoholism screening in patients with advanced cancer: impact on symptom burden and opioid use. J Palliat Med 11(7):964-968

29. Basch E, Prestrud AA, Hesketh PJ, Kris MG, Feyer PC, Somerfield MR, Chesney M, Clark-Snow RA, Flaherty AM, Freundlich B, Morrow G, Rao KV, Schwartz RN, Lyman GH (2011) Antiemetics: American Society of Clinical Oncology clinical practice guideline update. J Clin Oncol 29(31):4189-4198

30. Feyer PC, Maranzano E, Molassiotis A, Roila F, Clark-Snow RA, Jordan K (2011) Radiotherapy-induced nausea and vomiting (RINV): MASCC/ESMO guideline for antiemetics in radiotherapy: update 2009. Support Care Cancer 19(Suppl 1):S5-S14

31. Cherny NI, Portenoy RK (1994) The management of cancer pain. CA Cancer J Clin 44(5):263-303
32. Joly F, Vardy J, Pintilie M, Tannock IF (2007) Quality of life and/ or symptom control in randomized clinical trials for patients with advanced cancer. Ann Oncol 18(12):1935-1942

33. Farrar JT, Portenoy RK, Berlin JA, Kinman JL, Strom BL (2000) Defining the clinically important difference in pain outcome measures. Pain 88(3):287-294

34. Farrar JT, Young JP Jr, LaMoreaux L, Werth JL, Poole RM (2001) Clinical importance of changes in chronic pain intensity measured on an 11-point numerical pain rating scale. Pain 94(2):149-158

35. Ang SK, Shoemaker LK, Davis MP (2000) Nausea and vomiting in advanced cancer. Am J Hosp Palliat Care 27(3):219-225

36. Bruera E, Higginson IJ, Ripamonti C, von Guten C (2009) Textbook of palliative medicine. Hodder Arnold, London

37. Doyle D, Hanks G, Cherny N, Calman K (2011) Oxford textbook of palliative medicine. Oxford University Press, New York

38. Rhondali W, Nguyen L, Palmer L, Kang DH, Hui D, Bruera E (2013) Self-Reported Constipation in patients with advanced cancer: a preliminary report. J Pain Symptom Manage 45(1):23-32

39. Bruera E, Neumann C, Brenneis C, Quan H (2000) Frequency of symptom distress and poor prognostic indicators in palliative cancer patients admitted to a tertiary palliative care unit, hospices, and acute care hospitals. J Palliat Care 16(3):16-21

40. Glare PA, Dunwoodie D, Clark K, Ward A, Yates P, Ryan S, Hardy JR (2008) Treatment of nausea and vomiting in terminally ill cancer patients. Drugs 68(18):2575-2590

41. Lorusso V, Spedicato A, Petrucelli L, Saracino V, Giampaglia M, Perrone T (2009) Single dose of palonosetron plus dexamethasone to control nausea, vomiting and to warrant an adequate food intake in patients treated with highly emetogenic chemotherapy (HEC). Preliminary results. Support Care Cancer 17(12):1469-1473

42. Gomez-Batiste X, Porta-Sales J, Espinosa-Rojas J, Pascual-Lopez A, Tuca A, Rodriguez J (2010) Effectiveness of palliative care services in symptom control of patients with advanced terminal cancer: a spanish, multicenter, prospective, quasi-experimental, pre-post study. J Pain Symptom Manage 40(5):652-660

43. Delgado-Guay M, Yennurajalingam S, Parsons H, Palmer JL, Bruera E (2011) Association between self-reported sleep disturbance and other symptoms in patients with advanced cancer. J Pain Symptom Manage 41(5):819-827

44. Bruera E, Fadul N (2009) Constipation and diarrhea. In: Bruera E, Higginson IJ, Ripamonti C, von Guten C (eds) Textbook of palliative medicine. Hodder Arnold, London, pp 554-570

45. Glare P, Miller J, Nikolova T, Tickoo R Treating nausea and vomiting in palliative care: a review. Clin Interv Aging $6: 243-259$ 